\title{
Imprinting skyrmions in thin films by ferromagnetic and superconducting templates
}

Nuria Del-Valle, Sebastià Agramunt-Puig, Alvaro Sanchez, and Carles Navau

Citation: Appl. Phys. Lett. 107, 133103 (2015);

View online: https://doi.org/10.1063/1.4932090

View Table of Contents: http://aip.scitation.org/toc/apl/107/13

Published by the American Institute of Physics

\section{Articles you may be interested in}

Room temperature skyrmion ground state stabilized through interlayer exchange coupling

Applied Physics Letters 106, 242404 (2015); 10.1063/1.4922726

The influence of the edge effect on the skyrmion generation in a magnetic nanotrack

AIP Advances 7, 025105 (2017); 10.1063/1.4976726

Skyrmion creation and annihilation by spin waves

Applied Physics Letters 107, 152411 (2015); 10.1063/1.4933407

Current-driven skyrmion motion along disordered magnetic tracks

AIP Advances 7, 056017 (2017); 10.1063/1.4975658

Geometrical and physical conditions for skyrmion stability in a nanowire

AIP Advances 5, 047141 (2015); 10.1063/1.4919320

Eigenmodes of Néel skyrmions in ultrathin magnetic films

AIP Advances 7, 055212 (2017); 10.1063/1.4983806

\section{Scilight}

Sharp, quick summaries illuminating the latest physics research

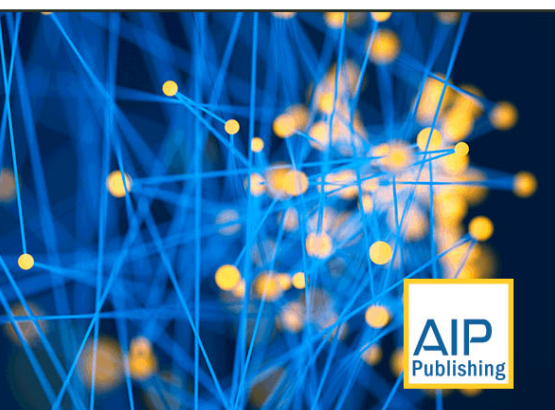




\title{
Imprinting skyrmions in thin films by ferromagnetic and superconducting templates
}

\author{
Nuria Del-Valle, Sebastià Agramunt-Puig, Alvaro Sanchez, and Carles Navau ${ }^{\text {a) }}$ \\ Departament de Física, Universitat Autònoma de Barcelona, 08193 Bellaterra, Barcelona, Catalonia, Spain
}

(Received 18 June 2015; accepted 19 September 2015; published online 29 September 2015)

\begin{abstract}
Magnetic skyrmions are promising candidates as information carriers in a new generation of memories. Here, we study the physical conditions for nucleating and stabilizing skyrmions by using local magnetic fields. We theoretically demonstrate how skyrmions can be imprinted in ultrathin ferromagnetic films, either individually and also in large numbers by bringing a magnetic nanostructured template close to the film. In this latter case, two kinds of templates, allowed by present-day nanotechnologies, are proposed: arrays of ferromagnetic nanorods or superconducting vortices. @ 2015 AIP Publishing LLC. [http://dx.doi.org/10.1063/1.4932090]
\end{abstract}

Skyrmion lattices of nanometer size have been observed in some magnetic materials. ${ }^{1}$ In particular, they have been obtained in two-dimensional films in the form of hexagonal swirling spin textures, stabilized at temperatures near zero ${ }^{2}$ or in the absence of magnetic field. ${ }^{3}$ A crucial factor in the stabilization of such lattices in magnetic metals lacking inversion symmetry is the interfacial Dzyaloshinskii-Moriya (DM) interaction. ${ }^{3-6}$ Magnetic films, such as Co layers on Pt, are excellent systems for studying and optimizing skyrmions. $^{7}$

Since electric currents much lower than those needed to move ferromagnetic domain walls can move skyrmions, 8,9 they have a high potential for using them as information carriers. ${ }^{10-12}$ Several works have studied the generation of skyrmions by local injection of currents, ${ }^{7,9,13,14}$ by attaching nanorods to the sample, ${ }^{15}$ or by nanodrilling the sample to obtain a skyrmion-like structure without DM interaction. ${ }^{16}$ Here, we propose an essentially different approach. We theoretically demonstrate an alternative way to nucleate and stabilize skyrmions: imprinting them using only local external magnetic fields. We show under which conditions induced skyrmions can be stable after removing the fields. Also, a further requirement for most applications is the controlled nucleation of a large number of skyrmions. We show that arrays of magnetic dipoles or monopoles, separated a distance of some tens of nanometers, can be used as nanomagnetic templates for imprinting a large number of skyrmions (Fig. 1). We discuss how nanotemplates involving either ferromagnetic rods or superconducting vortices fabricated with present nanotechnologies can be used.

To achieve our goals, we will first study the nucleation and stabilization of a single skyrmion by applying a local external magnetic field pulse. We will next study the conditions for generating and stabilizing an array of skyrmions using the external field coming from the aforementioned nanotemplates.

Our calculations are based on the dynamic LandauLifshitz-Gilbert equation, which can be written in dimensionless variables as

\footnotetext{
${ }^{a)}$ Electronic mail: carles.navau@uab.cat
}

$$
\left(1+\alpha^{2}\right) \frac{\mathrm{d} \mathbf{m}}{\mathrm{d} \tau}=-\mathbf{m} \times \mathbf{h}_{\mathrm{eff}}-\alpha \mathbf{m} \times\left(\mathbf{m} \times \mathbf{h}_{\mathrm{eff}}\right),
$$

where $\mathbf{m}$ is the magnetization vector and $\mathbf{h}_{\text {eff }}$ the effective field, both normalized to the saturation magnetization $M_{s}, \tau$ is the time normalized to $t_{0}=1 /\left(\gamma M_{s}\right), \gamma$ is the gyromagnetic factor, and $\alpha=\lambda M_{s} / \gamma$, where $\lambda$ is the damping constant. We consider the continuous approximation with the film lying on the $z=0$ plane (thickness is much smaller than any other relevant dimension). The normalized effective field includes the exchange, DM for isotropic ultrathin films, ${ }^{17}$ uniaxial anisotropy with the $z$ easy axis, and Zeeman interactions. Respectively,

$$
\mathbf{h}_{\text {eff }}=\nabla^{2} \mathbf{m}+\xi\left[(\nabla \mathbf{m}) \hat{z}-\nabla m_{z}\right]+\kappa m_{z} \hat{z}+\mathbf{h}_{\mathrm{a}},
$$

where all lengths are normalized to the exchange length $l_{\mathrm{ex}}=\sqrt{2 A /\left(\mu_{0} M_{s}^{2}\right)}$ being $A$ the exchange constant and $\mu_{0}$ the vacuum permeability. In the above equations, $\xi=D l_{\text {ex }} / A=D \sqrt{2 /\left(\mu_{0} M_{s}^{2} A\right)}$, being $D$ the DM-interaction constant, $\kappa=2 K /\left(\mu_{0} M_{s}^{2}\right)$ with $K$ the uniaxial anisotropy constant, and $\mathbf{h}_{\mathrm{a}}$ the applied field in units of $M_{s}$. Magnetostatic energy is disregarded for the present ultrathin films. ${ }^{7}$ This can be done because demagnetizing field depends linearly on thickness, whereas all other effective fields are independent on thickness (see numerical evaluation below). We do not consider temperature effects for simplicity. Periodic boundary conditions are used, considering that the template extends well beyond the calculation window.
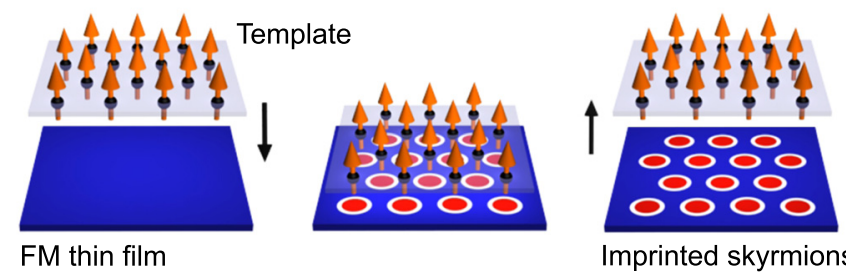

Imprinted skyrmions

FIG. 1. Sketch of the proposed procedure for imprinting skyrmions. A magnetic template is approached to an initially ferromagnetic (FM) thin film (left); under the appropriate conditions, a lattice of skyrmions is nucleated (center) and stabilized when the template, which remains unaffected, is removed away (right). 
To imprint a DM driven skyrmion in an initially ferromagnetic thin film, one has to overcome the topological barrier. We achieve this by applying a local magnetic field. An isolated magnetic moment pointing in the $-\hat{z}$ direction remains unstably static under the action of an external magnetic field $h_{a}$ in the positive $\hat{z}$ direction. However, a small deviation of the magnetic moment or a slight misalignment of the field would trigger a precession of the local magnetization around the applied field direction and a tendency to align in that direction. The flipping time would be inversely proportional to the applied field and to the damping coefficient. If this magnetic moment is also affected by uniaxial anisotropy, there would be a threshold applied field to trigger the flip $h_{a} \sim \kappa$. In the present case, however, we do not have isolated magnetic moments but a continuous magnetization that can be viewed as moments interacting with their neighbors through both exchange and DM interactions. Thus, the neighboring moments would also affect the flipping time. Actually, the exchange interaction would fight against the flip, whereas the DM interaction would produce some angular components of the magnetization, helping the precession and accelerating the flip. As a result, a strong enough (for beating the anisotropy) local non-uniform field (for providing some angular components), applied during enough time (sufficient for flipping) can trigger the generation of a skyrmion.

In order to quantify these ideas, we show in Fig. 2 the calculated temporal evolution of the magnetic structures created in an ultrathin film considering different values of $\kappa$ and different types of sources for the local external field. We plot the $m_{z}$ component of the magnetization distribution. To fix ideas, we consider $\alpha=0.3, \xi=1.68$ and that either a dipole (Fig. 2, left) or a monopole (Fig. 2, right) is sinusoidally approaching and retreating from the film during a time of 512 units of $t_{0}$. The normalized distance from the source to the film is $\delta$. When the external magnetic source is at the largest distance, the field is basically negligible, and different lowermost $\delta$ 's are considered. We use a normalized magnetic moment of the dipole $m_{d} /\left(M_{s} l_{\mathrm{ex}}^{3}\right)=902$ and a normalized magnetic charge of the monopole $Q /\left(M_{s} l_{\mathrm{ex}}^{2}\right)=39$. All these numbers correspond to realistic values when denormalized, as shown below.

Results show that ring-shaped structures are nucleated when the anisotropy is small (Fig. 2, $\kappa=0$ columns), similar to those in Ref. 18 and to some larger ones in Refs. 19 and 20 without DM interaction. These structures arise from the coordinate flip of the moments at several radial distances, produced by the radial components of the applied field helped by the DM interaction [rightmost term in Eq. (1)]. When $\kappa$ increases, radial components are not large enough until $\delta$ has decreased and, at that moment, radial components are intense close to the center of the film, producing a single bump in the vertical magnetization, instead of a ring-shaped one (Fig. 2, $\kappa>0$ columns, except $\kappa=2$ for the monopolar source). The calculated skyrmionic number $N_{s}=$ $(1 / 4 \pi) \int \mathbf{m} \cdot\left(\frac{\partial \mathbf{m}}{\partial x} \times \frac{\partial \mathbf{m}}{\partial y}\right) \mathrm{d} x \mathrm{~d} y$ when a central bump is formed grows from zero to a fixed value that is basically 1 for low anisotropies and decreases when anisotropy increases. This confirms the overcoming of the topological barrier.

Figure 2 also shows that the anisotropy delays the formation (and evolution) of structures. It can even be a preventing factor for the nucleation of skyrmions, as seen in the rightmost column in Fig. 2, where the monopolar field has not been strong enough to trigger the skyrmion nucleation, although the same field does it when $\kappa$ is smaller. For the dipolar source, no nucleation occurs for $\kappa \geqq 7$.

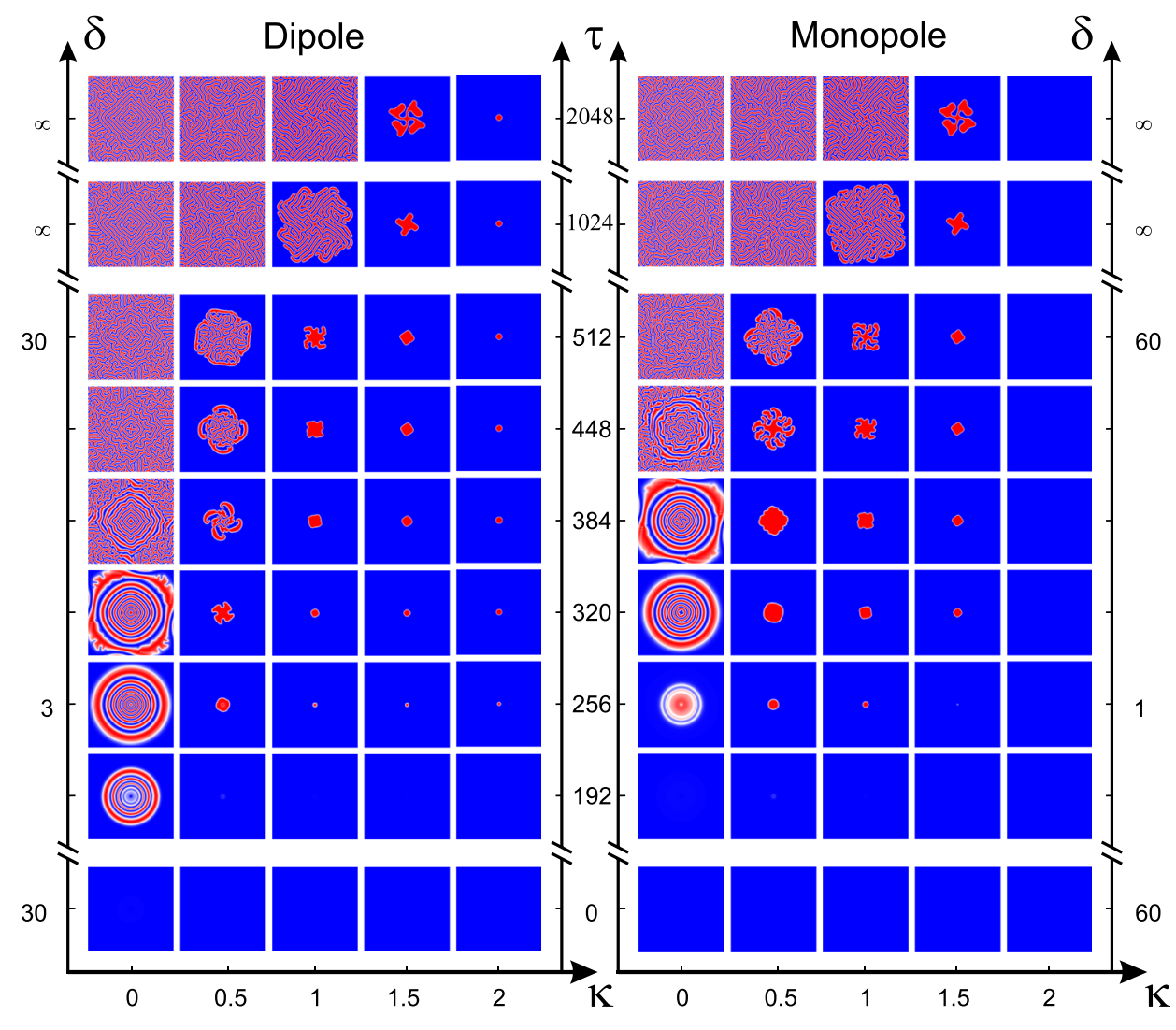

FIG. 2. Temporal evolution (normalized time $\tau$ is in the central vertical axis) of the $m_{z}$ distribution (blue, white, red indicate $+1,0,-1$, respectively) of the magnetic structures created when a dipolar (left set of figures) and a monopolar (right set of figures) source is approaching-retreating from the film. Left and right vertical axis indicate the normalized distances corresponding to the central times, considering a sinusoidal function up to $\tau=512$. For $\tau>512$, the distance is set to infinite. Each figure corresponds to a squared calculation window of $200 \times 200$ (in units of $l_{\mathrm{ex}}$ ). Different columns indicate calculations using different values of the normalized anisotropy constant. We have used $\alpha=0.3, \xi=1.68, m_{d} /\left(M_{s} l_{\mathrm{ex}}^{3}\right)=902$, and $Q /\left(M_{s} l_{\mathrm{ex}}^{2}\right)=39$. 
As time evolves, the imprinted magnetic structures with low anisotropy tend to become larger. Eventually, new skyrmions or rings can appear inside the original ring (Fig. 2, $\kappa=0$ columns) and, after some time, the structure evolves to a worm-like pattern (Fig. 2, $\kappa=0$ columns, $\tau \geq 384$ rows), as observed in Refs. 21 and 22. When the central skyrmion is formed, the presence of anisotropy delays the formation of such complex structures. Interestingly, there are situations in which the anisotropy is not large enough to prevent nucleation but can fully stabilize the already nucleated skyrmion (Fig. 2, $\kappa=2$ column for dipolar source). This can be understood from a rough argument. Consider an idealized skyrmion of radius $R$, where $m_{z}=\left(R^{2}-\rho^{2}\right) /\left(R^{2}+\rho^{2}\right)$ and $m_{\rho}=\sqrt{1-m_{z}^{2}}$, being $\rho$ the radial distance from the center of the skyrmion. ${ }^{23}$ The exchange energy is independent of the radius $R$, whereas the DM energy is negative and depends linearly upon $R$. Thus, the skyrmion, in the absence of other energies, will tend to increase its radius. The anisotropy energy is positive and has basically a $R^{2}$ dependence. Thus, the growth of the skyrmion radius can be stopped since there can be a minimum in the total energy versus radius function. For large radius, it is more energetically favorable to have several oscillations in the $m_{z}$ distribution than a single central bump, and the magnetic structure tends to a largely oscillating distribution. The presence of anisotropy changes the shape of the magnetic structure, trying to reduce the regions where $m_{z} \neq \pm 1$. The exact dependencies of the energies on the radius change with respect to the idealized skyrmion, but the overall behavior still remains.

Anisotropy is not the only way of stabilizing skyrmions. Once formed, a uniform external field, with an adequate value, can stabilize skyrmions ${ }^{6,11}$ (the dependence of the Zeeman energy upon the radius $R$ of an idealized skyrmion is $\sim R^{2}$ ). Here, we propose an alternative stabilization mechanism by using the presence of other skyrmions created by local fields at different positions. The idea is to use the topological barrier between neighboring structures to mutually prevent their growth.

We now discuss the possible practical implementation of these ideas. We consider a magnetic film with parameters $M_{s}=580 \mathrm{kA} \mathrm{m}^{-1}, A=15 \mathrm{pJ} \mathrm{m}^{-1}$, and $D=3 \mathrm{~mJ} \mathrm{~m}^{-2}$, corresponding to perpendicularly magnetized Co layers on $\mathrm{Pt}^{7}$ The uniaxial anisotropy constant will be varied up to $K=0.6 \mathrm{MJ} \mathrm{m}^{-3}$ in the calculations. ${ }^{7,24}$ Several techniques can be used for modifying $K{ }^{25}$ We use $\alpha=0.3$ (Ref. 24) and $\gamma=2.21 \times 10^{5} \mathrm{~m} \mathrm{~A}^{-1} \mathrm{~s}^{-1}$, which gives $t_{0}=7.8 \times 10^{-12} \mathrm{~s}$ (so $1 \mathrm{~ns}$ is $128 t_{0}$ ), $l_{\mathrm{ex}}=8.4 \mathrm{~nm}, \xi=1.68$, and $\kappa=0-2.84$, justifying the values used in the dimensionless analysis above. Using these values, and considering a thickness of $0.4 \mathrm{~nm},{ }^{7}$ the exchange and the DM effective fields are of the order of a few Tesla, whereas the demagnetizing field is at least two orders of magnitude less, justifying neglecting the demagnetizing energy in the calculations.

We consider a template formed by a hexagonal array with lattice constant $a$ of two possible types. (i) A dipolarlike one, which could be constructed using magnetic tips. We consider an effective magnetic moment for each dipole of $m_{d}=3.1 \times 10^{-16} \mathrm{~A} \mathrm{~m}^{2}$ (Ref. 26) $\left[m_{d} /\left(M_{s} l_{\mathrm{ex}}^{3}\right) \simeq 902\right]$. The dipole is approached to the magnetic film sinusoidally from $250 \mathrm{~nm}$ down to $25 \mathrm{~nm}\left(\simeq 3 l_{\mathrm{ex}}\right)$ during a time of $2 \mathrm{~ns}$ $\left(256 t_{0}\right)$, a typical time scale for structure formation ${ }^{7}$ (although these values are demanding in terms of practical applications involving large velocities, larger exposition times do not substantially modify the qualitative behavior). (ii) A monopolar-like template formed by an array of superconducting vortices. ${ }^{27}$ At distances of the order of the Pearl penetration depth (typically, tens of nanometers), a vortex in a thin superconductor creates to a good approximation a monopolar field. ${ }^{28,29}$ We consider an array of superconducting vortices, each with the charge corresponding to one fluxoid per site, $Q=\Phi_{0} / \mu_{0}=1.6 \times 10^{-9}$ A $\mathrm{m}\left(\Phi_{0}\right.$ is the magnetic flux quantum) $\left[Q /\left(M_{s} l_{\mathrm{ex}}^{2}\right) \simeq 39\right]$. This template can be tailored with separations of few tens of nanometers (few $l_{\text {ex }}$ 's) by having an array of antidots in superconducting films where a single fluxoid is present in each antidot. This template is sinusoidally descended from $500 \mathrm{~nm}$ to $8.4 \mathrm{~nm}$ $\left(\simeq 1 l_{\mathrm{ex}}\right)$ during a time of $2 \mathrm{~ns}$. When both templates are at their lowest distance, the field created by one source (either dipole or monopole) is of the order of $2 \mathrm{~T}$, which corresponds to the value $h_{a} \simeq \kappa$ (at the starting point the field is negligible). These realistic numbers justify the values used above.

We show in Fig. 3 the $m_{z}$ magnetization distribution in films with $K=0,0.3$, and $0.6 \mathrm{MJ} \mathrm{m}^{-3}$, evaluated after $16 \mathrm{ns,}$ well after the template has been approached, the magnetic structures have been imprinted and the template is retreated back (after $8 \mathrm{~ns}$ the external field is negligible). All the shown structures are stable after this time. For the case of a dipolar template, we observe (Fig. 3, up, $K \neq 0$ cases) how a skyrmion lattice (as in Ref. 14, for example) can be imprinted and stabilized forming a regular lattice mimicking the template lattice. The skyrmions have a diameter $\simeq 25 \mathrm{~nm}$. When the anisotropy is not strong enough to stabilize single skyrmions (Fig. 3, up, $K=0$ ), structures can be formed depending on the $a$ parameter of the template. When the sources are too close to each other, the flip of the magnetization below the sources is greatly affected by the presence of other sources and, in fact, not a single site but a chain of sites flip simultaneously their magnetization. The subsequent time evolution produces an oscillating structure similar to that observed in Refs. 19 and 11. If $a$ is large, there is room for the different skyrmions to evolve and distort, and form some structure before meeting the neighboring structures. For $a=75 \mathrm{~nm}$, we find a regular array of quasicircular bubbles (similar to those observed in Ref. 15, for example). We note, however, that this state could be unstable and small perturbations (because of temperature or some inhomogeneity) will break the configuration to a strip-like helical less energetic state.

In the case of the monopolar sources (Fig. 3, down), the situation is similar although, for the $K=0.6 \mathrm{MJ} \mathrm{m}^{-3}$ case, the field created by the monopolar template is not enough to nucleate structures. For the $K=0.3 \mathrm{MJ} \mathrm{m}^{-3}$ case, the bubbles are stabilized due to the neighbors, since the anisotropy is not strong enough to freeze their expansion. When $a$ is too small, the large applied field produces a complete change in the magnetization of the film and no structure is formed. In this case, the range of adequate $K^{\prime} s$ (or, equivalently, for a given $K$, the range of adequate distances) for stabilizing a skyrmion lattice is reduced with respect to that of the dipolar case. 

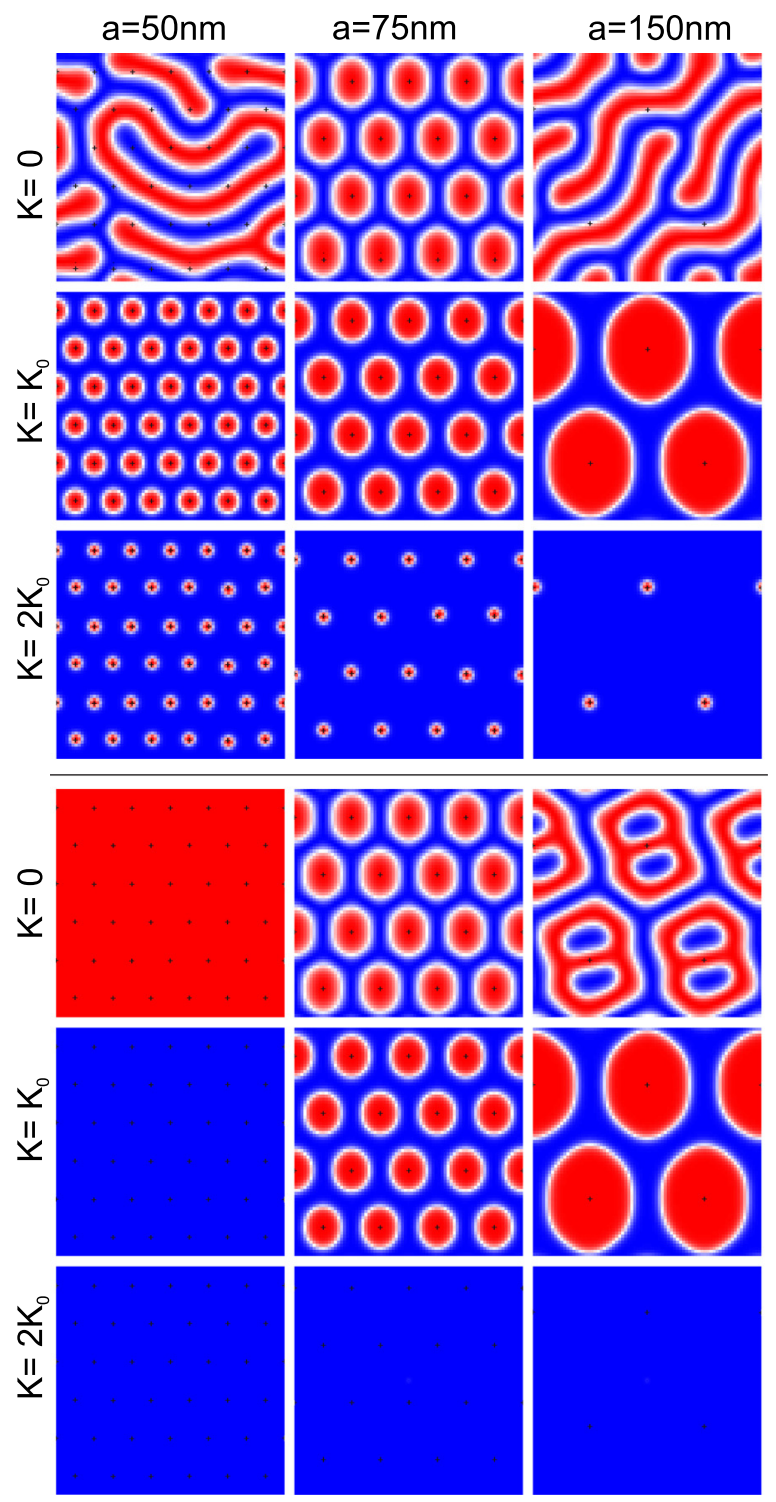

FIG. 3. Distribution of $m_{z}$ when arrays of dipoles (three upper rows) or monopoles (three lower rows) with different $a$ 's (columns) are used as templates. The picture shows the distribution well after the template has been removed. We show, for each template, three cases corresponding to different possible anisotropy constants when $K_{0}=0.3 \mathrm{MJ} \mathrm{m}^{-3}$. Each figure corresponds to a squared calculation window of $300 \times 300 \mathrm{~nm}^{2}$. We have used $M_{s}=580 \mathrm{kA} \mathrm{m}^{-1}, A=15 \mathrm{pJ} \mathrm{m}^{-1}, D=3 \mathrm{~mJ} \mathrm{~m}^{-2}, \alpha=0.3, \gamma=2.21 \times 10^{5}$ $\mathrm{m} \mathrm{A}^{-1} \mathrm{~s}^{-1}, m_{d}=3.1 \times 10^{-16} \mathrm{~A} \mathrm{~m}^{2}$, and $Q=1.6 \times 10^{-9} \mathrm{~A} \mathrm{~m}$. Colors are the same as in Fig. 2.

The stray field corresponding to the generated skyrmion structures is about $2 \mathrm{mT}$ at $25 \mathrm{~nm}$, well below the coercivity of typical arrays of magnetic nanowires (around $15 \mathrm{mT},{ }^{30,31}$ and about $5 \mathrm{mT}$ at $10 \mathrm{~nm}$, well below the field typically needed to depin superconducting vortices in arrays of antidots (around $20 \mathrm{mT}$ for a typical $\mathrm{Pb}$ film ${ }^{32}$ ). Thus, the proposed magnetic templates would not be demagnetized by the skyrmion's stray fields and could be reused several times. One could even envisage a manufacturing chain of skyrmion lattices in this way.

To conclude, we have shown how local magnetic fields can be used to overcome the topological barrier for nucleating skyrmions. Two ways of stabilizing the skyrmions after removing the external field have been demonstrated: by magnetic anisotropy and by the presence of neighboring skyrmions forming a regular array. Templates of nanometer-scale magnetic objects can be used for imprinting arrays of skyrmions in magnetic films, after exposure times as short as few nanoseconds. These results hint at the possibility of using these ideas as a fast way to write large quantities of nanomagnetic elements, which could be manipulated and read through the free surface of the film, for a future generation of magnetic memories.

The authors thank Catalan projects 2014SGR150 and 2014PROD00059, Spanish project MAT2012-35370, and FEDER for financial support. A.S. also thanks financial support from ICREA Academia (Generalitat de Catalunya).

${ }^{1}$ S. Mühlbauer, B. Binz, F. Jonietz, C. Pfleiderer, A. Rosch, A. Neubauer, R. Georgii, and P. Böni, Science 323, 915 (2009).

${ }^{2}$ X. Z. Yu, Y. Onose, N. Kanazawa, J. H. Park, J. H. Han, Y. Matsui, N. Nagaosa, and Y. Tokura, Nature 465, 901 (2010).

${ }^{3}$ U. K. Rössler, A. N. Bogdanov, and C. Pfleiderer, Nature 442, 797 (2006).

${ }^{4}$ A. Bogdanov and A. Hubert, J. Magn. Magn. Mater. 138, 255 (1994).

${ }^{5}$ A. Bogdanov and U. Röß ler, Phys. Rev. Lett. 87, 037203 (2001).

${ }^{6}$ J. H. Han, J. Zang, Z. Yang, J.-H. Park, and N. Nagaosa, Phys. Rev. B 82, 094429 (2010).

${ }^{7}$ J. Sampaio, V. Cros, S. Rohart, A. Thiaville, and A. Fert, Nat. Nanotechnol. 8, 839 (2013).

${ }^{8}$ F. Jonietz, S. Mühlbauer, C. Pfleiderer, A. Neubauer, W. Münzer, A. Bauer, T. Adams, R. Georgii, P. Böni, R. A. Duine et al., Science 330, 1648 (2010).

${ }^{9}$ S.-Z. Lin, C. Reichhardt, C. D. Batista, and A. Saxena, Phys. Rev. B 87, 214419 (2013).

${ }^{10}$ A. Fert, V. Cros, and J. a. Sampaio, Nat. Nanotechnol. 8, 152 (2013).

${ }^{11}$ N. Nagaosa and Y. Tokura, Nat. Nanotechnol. 8, 899 (2013).

${ }^{12}$ R. Tomasello, E. Martinez, R. Zivieri, L. Torres, M. Carpentieri, and G. Finocchio, Sci. Rep. 4, 6784 (2014).

${ }^{13}$ Y. Tchoe and J. H. Han, Phys. Rev. B 85, 174416 (2012).

${ }^{14}$ N. Romming, C. Hanneken, M. Menzel, J. E. Bickel, B. Wolter, K. von Bergmann, A. Kubetzka, and R. Wiesendanger, Science 341, 636 (2013).

${ }^{15}$ L. Sun, R. X. Cao, B. F. Miao, Z. Feng, B. You, D. Wu, W. Zhang, A. Hu, and H. F. Ding, Phys. Rev. Lett. 110, 167201 (2013).

${ }^{16}$ M. V. Sapozhnikov and O. L. Ermolaeva, Phys. Rev. B 91, 024418 (2015).

${ }^{17}$ S. Rohart and A. Thiaville, Phys. Rev. B 88, 184422 (2013).

${ }^{18}$ A. O. Leonov and M. Mostovoy, Nat. Commun. 6, 8275 (2015).

${ }^{19}$ M. Hehn, K. Ounadjela, J.-P. Bucher, F. Rousseaux, D. Decanini, B. Bartenlian, and C. Chappert, Science 272, 1782 (1996).

${ }^{20}$ M. Hehn, S. Padovani, K. Ounadjela, and J. Bucher, Phys. Rev. B 54, 3428 (1996).

${ }^{21}$ C. Moreau-Luchaire, C. Moutafis, N. Reyren, J. Sampaio, N. Van Horne, C. A. F. Vaz, K. Bouzehouane, K. Garcia, C. Deranlot, P. Warnicke et al., preprint arXiv:1502.07853 (2015).

${ }^{22}$ X. Yu, M. Mostovoy, Y. Tokunaga, W. Zhang, K. Kimoto, Y. Matsui, Y. Kaneko, N. Nagaosa, and Y. Tokura, Proc. Natl. Acad. Sci. U. S. A. 109, 8856 (2012).

${ }^{23}$ A. Kirakosyan and V. Pokrovsky, J. Magn. Magn. Mater. 305, 413 (2006).

${ }^{24}$ P. Metaxas, J. Jamet, A. Mougin, M. Cormier, J. Ferré, V. Baltz, B. Rodmacq, B. Dieny, and R. Stamps, Phys. Rev. Lett. 99, 217208 (2007).

${ }^{25}$ C. Chappert, Science 280, 1919 (1998).

${ }^{26}$ H. Li, Y. Wang, S. Wang, H. Zhong, and D. Wei, IEEE Trans. Magn. 46, 2570 (2010).

${ }^{27}$ J. Pearl, Appl. Phys. Lett. 5, 65 (1964).

${ }^{28}$ J. Clem, Phys. Rev. B 43, 7837 (1991).

${ }^{29}$ O. Romero-Isart, C. Navau, A. Sanchez, P. Zoller, and J. I. Cirac, Phys. Rev. Lett. 111, 145304 (2013).

${ }^{30}$ E. Nikulina, O. Idigoras, P. Vavassori, A. Chuvilin, and A. Berger, Appl. Phys. Lett. 100, 142401 (2012).

${ }^{31}$ A. Fernández-Pacheco, L. Serrano-Ramón, J. M. Michalik, M. R. Ibarra, J. M. De Teresa, L. O'Brien, D. Petit, J. Lee, and R. P. Cowburn, Sci. Rep. 3, 1492 (2013).

${ }^{32}$ A. Silhanek, S. Raedts, M. Lange, and V. Moshchalkov, Phys. Rev. B 67, 064502 (2003). 